\title{
Analysis of postural stability for Chinese crews working on moving ocean platforms
}

\author{
Zhaofang Liu ${ }^{\mathrm{a}}$, Hong Lin \\ Food Science and Engineering College, Ocean University of China, 266100 Qingdao, China
}

\begin{abstract}
Moving ocean platforms, such as ship, fishing vessels and offshore platforms etc, suffer seriously from sea loads as wind, wave, currents and other environmental factors. Platforms' motions induced from various sea loads have significant effects on crews aboard. Severe motions of the platform can make crew lose balance or cause motion induced interruptions (MIIs). This paper studies postural stability of crews on moving platforms based on Chinese anthropometric standards. The relationships between height of center of mass (COM), moment of inertia and human's height, mass of human body are analysed. The postural stability indicator, tipping coefficient is obtained of human body on moving platform by applying Chinese anthropometric standards. Then the stability domains of human bodies with translational is derived considering the physical parameters of Chinese adults. The work can be helpful for estimation of MII probability and safety of crews on moving ocean platform.
\end{abstract}

\section{Introduction}

Floating platforms are widely used in marine transportation, fishery, exploitation of ocean resources and oceanographic survey. Due to the loads of wind, wave and current of ocean environment, motions of ocean platforms in six degree of freedom (sway, surge, heave, pitch, roll and yaw) occur frequently. These motions have significant effects on the health and safety of crews aboard. Motions of ocean platforms have various negative effects, such as Motion Induced Interruptions (MIIs), Motion Induced Fatigue (MIF) and Motion Sickness (MS) to human(Wertheim,1998) ${ }^{[1]}$. MIIs were defined as the occasions when sailors on ships with strong motion are easily to become unstable or lose balance and have to stop their work in order to avoid further damage(Graham,1990) ${ }^{[2]}$. In China, there are huge number of workers as sailors, fishermen, and offshore crews working on moving floating ocean platforms. All the people mentioned above are facing serious health and safe hazards from these motions.

There is plenty of research work on human motion on moving platforms. Graham (1990) ${ }^{[2]}$ made the definition of MII and pointed out it had obvious effects on comfort and performance of human aboard. He developed the model for MII with simplified physical parameters of human body. It provided a useful way to quantify human performance on ships and predicted the number of motioninduced interruptions. Crossland used the model to compare the number of MIIs between experiment and estimation, and found they agreed fairly well(Crossland, 1998 $)^{[3]}$.

\footnotetext{
${ }^{\mathrm{a}}$ Corresponding author : lzf@ouc.edu.cn
} 
Haward, Lewis and Griffin(2009) ${ }^{[4]}$ studied the whole human vibration under the motion of platforms and analysed the relationship between response and factors such as exciting frequency, amplitude, direction and so on. Nawayseh and Griffin $\left((2006)^{[5]}\right.$ studied the effect of frequency, magnitude and direction of oscillation on the postural stability of standing people. Complex models as articulated inverse pendulum model were also adopted to study the control of human body(Wedge and Langlois,2003) ${ }^{[6]}$. Yao et al studied the human response on ocean platform based on the multi-body dynamics model ${ }^{[7]}$. An review of the state of art on postural of human body on moving platform was given (Erica Nocerino, 2010) ${ }^{[8]}$. Based on existing research some guide rules were given in ABS for habitability of crew on moving platform or offshore installation (ABS, 2010) ${ }^{[9]}$.

Most of the existing MII models neglect the actual physical parameters of human body, especially the variation of people from different countries. In order to evaluate human performance accurately, this paper studies the stability domains of human on moving ocean platform. Physical parameters of Chinese crews are considered based on national standard of Chinese adult anthropometry. Then, stability domains of human could be obtained by solving equations of the MII model.

\section{Human parameters based on Chinese standard}

The three-dimension human body model and anatomical planes are shown in Figure 1(a). In this paper only the motion in coronal plane is studied and the coordinate frames of human body O-XY is shown in Figure 2(b) and the origin point locates on the gravity centre of human body.

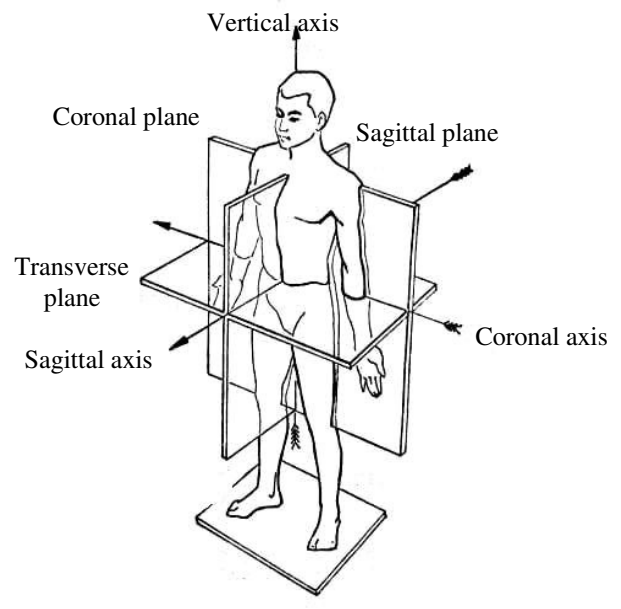

(a) Basic planes and axises in human body model

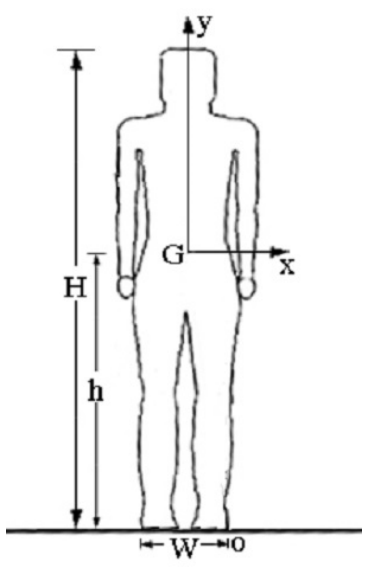

(b) Coordinate system of human body

Figure 1. Scheme of human body.

Basic planes and axes in human body model are shown in Fig 1(a). In this paper only the motion in coronal plane is studied (Fig 1(a)); and the coordinate frames of human body O-XY is shown in Fig 2(b), and the origin point locates on the gravity centre of human body.

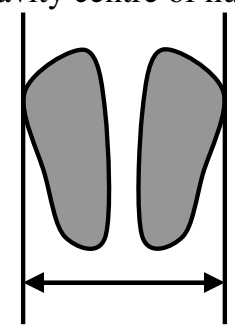

Figure 2. Feet width with natural standing. 
Dimension parameters and physical parameters of human body have an important role on postural stability such as height of COM, weight and inertia of moment of human body et al. Different postures can also have a significant effect on stability. These parameters are closely related to the factors as nation, age, gender and occupations.

Width of feet, dimension and area of feet occupying et al can also affect the postural stability obviously. In this paper only natural standing posture is considered, which is standing up straight with feet about shoulder width apart. According to convention of ergonomics, the width of feet or shoulder width $W$ (shown in Figure 2) is a function of the height of human body $H$ as

$$
W=0.222 H
$$

As to Chinese, several national standards on human factors have been carried out such as GB 10000-1988[16], GB/T 12985-1991[17] and GB/T 17245-2004[13]. In the latter standard the values of average height of $\mathrm{COM}$, moment of inertia along with standard abbreviation are given. Moreover, the standard also provided function reflecting the relationship between height of COM, inertia of moment with mass, height and so on based on Chinese anthropometry data. The relationship between height of mass centre and body mass, height is:

$$
h=H-\left(B_{0}+B_{1} m+B_{2} H\right)
$$

Where $m$ is the mass of human body, $H$ is the height of human body, $B_{0} 、 B_{1}$ and $B_{2}$ are regressive coefficients. The coefficients for male and female are shown in Table 1.

Table 1. Regressive coefficients of height of COM of Chinese adults.

\begin{tabular}{|c|c|c|c|c|}
\hline Gender & $\begin{array}{c}\text { Constant term } \\
B_{0}\end{array}$ & $\begin{array}{c}\text { Coefficient of mass } \\
B_{1}\end{array}$ & $\begin{array}{c}\text { Coefficient of height } \\
B_{2}\end{array}$ & $\begin{array}{c}\text { Multiple correlation } \\
\text { coefficient } R\end{array}$ \\
\hline Male & -32.2975 & -0.4443 & 0.478 & 0.833 \\
\hline Female & -95.1467 & -0.5457 & 0.5311 & 0.921 \\
\hline \multicolumn{2}{rl}{ Remark: Unit of mass is kg, unit of height is mm. } \\
\hline
\end{tabular}

According to equation (2), values of the height of COM calculated by Graham's model ${ }^{[2]}$ and the present model are shown in Figure 3. Figure 3(a) shows that height of COM increases with the height of human body when the mass is constant value $(60 \mathrm{Kg})$. Figure $3(\mathrm{~b})$ shows that the height of COM increases with the mass when the height is constant value as $1700 \mathrm{~mm}$. In this figure, the height of COM increases slightly with the mass in Chinese adult's model while it maintains constant in Graham's model. From these two figures, as a whole, it is clear that the height of mass centre is larger in Chinese adult's model than the model of Graham's model.

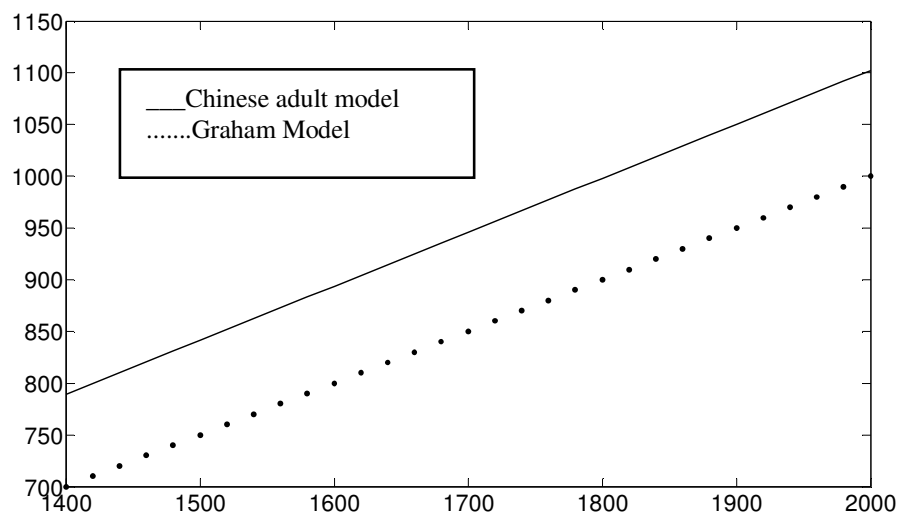

(a) 


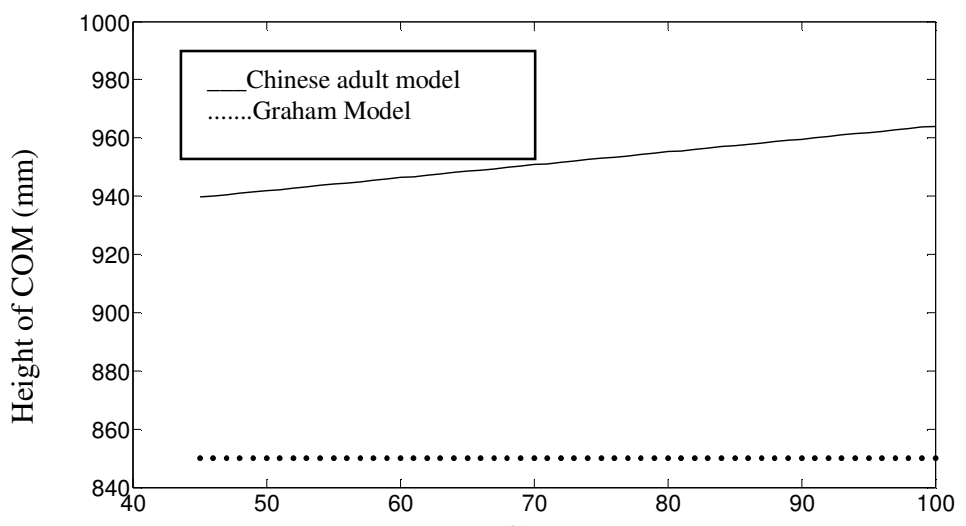

(b)

Figure 3. Relationship between Height of mass center and mass, height (a)human body with mass of 60kg, (b) human body with height of $1700 \mathrm{~mm}$.

According to Chinese adult's anthropometry standard (GB/T 17245-2004), the moment of inertia is related to mass and height as below:

$$
I_{i}=C_{0}+C_{1} m+C_{2} H \quad(i=x, y, z)
$$

Where $C_{0}, C_{1}$ and $C_{2}$ are the regressive coefficients. The coefficients of equation (3) can be found in Chinese adult's anthropometry standard (GB/T 17245-2004) ${ }^{[13]}$. For the moment of inertia with respect to COM on coronal plane the coefficients are shown in Table 2.

Table 2. The regressive coefficients of moment of inertia of Chinese adults ${ }^{[13]}$.

\begin{tabular}{|c|c|c|c|c|c|}
\hline Gender & $\begin{array}{l}\text { Moment of } \\
\text { inertia }\end{array}$ & $\begin{array}{l}\text { Constant term } \\
C_{0}\end{array}$ & $\begin{array}{l}\text { Coefficient of } \\
{ }_{\operatorname{mass}} C_{1}\end{array}$ & $\begin{array}{l}\text { Coefficient of } \\
\text { height } \\
C_{2}\end{array}$ & $\begin{array}{c}\text { Complex } \\
\text { coefficient } R\end{array}$ \\
\hline Male & $I_{G}$ & -25397472.8 & 130503.9 & 16396.1 & 0.971 \\
\hline Female & $I_{G}$ & -17309877.5 & 129946.20 & 11468.6 & 0.964 \\
\hline \multicolumn{6}{|c|}{$\begin{array}{l}\text { Remark: Unit of mass is } \mathrm{kg} \text {, unit of height is } \mathrm{mm} \text {, unit of inertia is } \mathrm{kg} \cdot \mathrm{mm}^{2} \cdot I_{G} \text { is moment of inertia about } \\
\text { sagittal axis through the COM. }\end{array}$} \\
\hline
\end{tabular}

Because motions in coronal plane are studied, moment of inertia about sagittal axis through foot edge point $O$ or $O$ ' is:

$$
I_{O}=I_{G}+m\left(w^{2}+h^{2}\right)
$$
COM.

Where $w$ is half of a person's feet width $W$ with the natural standing posture, $h$ is the height of

Based on equation (4), we can obtain the value of the moment of inertia about supporting foot edge point $\mathrm{O}$ in standing human body. It illustrates the relationship between moment of inertia $I_{O}$ and the mass/ height of human body in this model and Graham's model. Figure 4(a) shows that $I_{O}$ increases with the height of human body when the mass is constant value $(60 \mathrm{Kg})$. Figure 4 (b) shows that the height of COM varies as the mass increases when the height is constant value as $1700 \mathrm{~mm}$. From these two figures, it is clear that the moment of inertia ${ }^{\circ}$ is also larger in Chinese adult's model than the model of Graham's model. It should be noticed that the height and moment of inertia are 
closely related to the standing posture, especially the width of feet separated in the Chinese adult model in this paper.

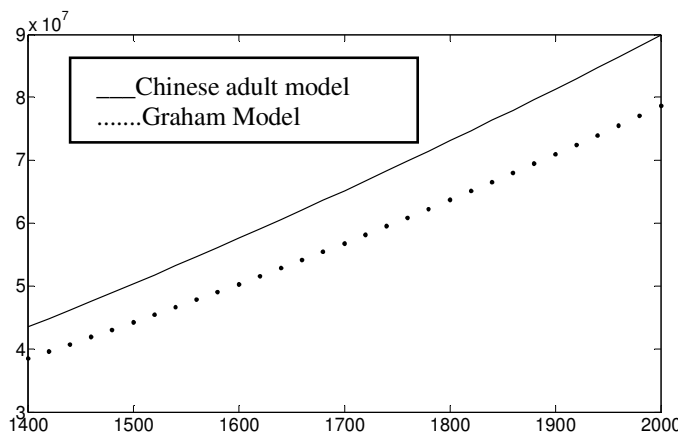

(a)

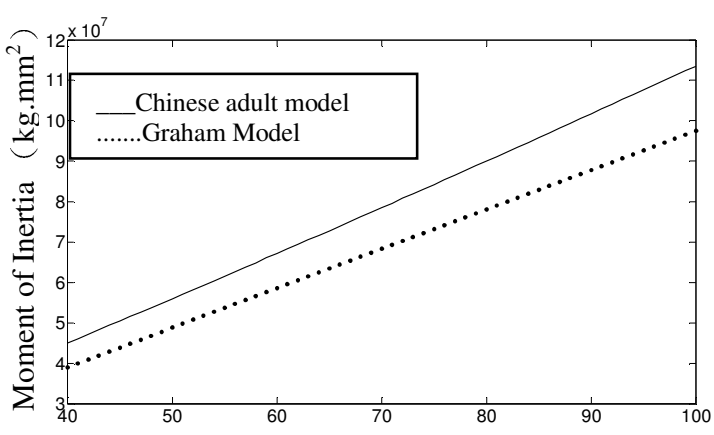

(b)

Figure 4. Relationship between Moment of inertia and height, mass of human body (a) human body with the mass of $60 \mathrm{~kg}$, (b) human body w ith the height of $1700 \mathrm{~mm}$.

\section{Human body model for Mlls estimation}

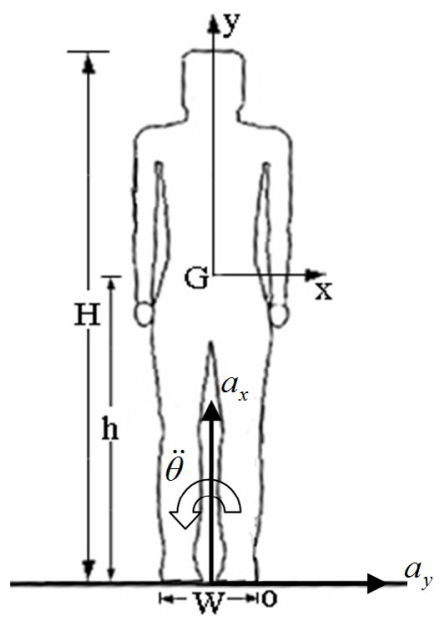

Figure 5. Standing human model.

The model for the analysis of stability of standing human with the translation and rotation excitation is shown in Figure 4. Given the large friction coefficient between boots bottom of crews and deck, the possibility of slip motion is neglected.

According to human on ocean platform suffering from the motions in translation about $\mathrm{x}, \mathrm{y}$ axis and rotation about $\mathrm{z}$, the motion equation of human body rotating about $\mathrm{O}$ can be described as:

$$
I_{o} \ddot{\theta}-F_{x} h-F_{y} w=0
$$

Where $\theta$ and $\ddot{\theta}$ are the angle and angular acceleration of human body,

$I_{O}$ is the moment of inertia about one foot,

$F_{x}$ is the total force on horizontal direction,

$F_{y}$ is the total force on vertical direction, 
$h$ is the height of a person' COM from the deck,

$w$ is the half of a person's standing width.

When $\theta$ is small enough, $F_{x} 、 F_{y}$ can be simplified into

$$
\begin{gathered}
F_{x}=m\left(a_{x}+g \sin (\theta)\right) \approx m\left(a_{x}+g \theta\right) \\
F_{y}=m\left(a_{y}+g \cos (\theta)\right) \approx m\left(a_{y}+g\right)
\end{gathered}
$$

The motion equation of human body can be obtained as:

$$
\frac{I_{O}}{m h} \ddot{\theta}-\left(a_{x}+g \theta\right)-\frac{w}{h} a_{y}>\frac{w}{h} g
$$

In this situation, the human body will tip to the left direction.

Similarly, when human body tips about point O', the motion equation is:

$$
-\frac{I_{O^{\prime}}}{m h} \ddot{\theta}+\left(a_{x}+g \theta\right)-\frac{w}{h} a_{y}>\frac{w}{h} g
$$

Human parameters in these equations have been calculated and can be applied in the MII prediction. If ocean platforms only have translational motion, then equation (8) could be amended as:

$$
-a_{x}-\frac{w}{h} a_{y}>\frac{w}{h} g
$$

Similarly, equation (9) could be amended as:

$$
a_{x}-\frac{w}{h} a_{y}>\frac{w}{h} g
$$

In equation (10) and (11), we can find that the ratio $\frac{w}{h}$ is an essential factor to affect the stability of human, which is defined as tipping coefficient $e$. It is obvious that probability of crews suffering from MII on ocean platform would increase with tipping coefficient. Based on equation (2), (12) and (13) the tipping coefficient is:

$$
e=\frac{w}{h}=\frac{0.111 H}{H-\left(B_{0}+B_{1} m+B_{2} H\right)}
$$

The relationship between tipping coefficient, height and mass is shown in Figure 6.

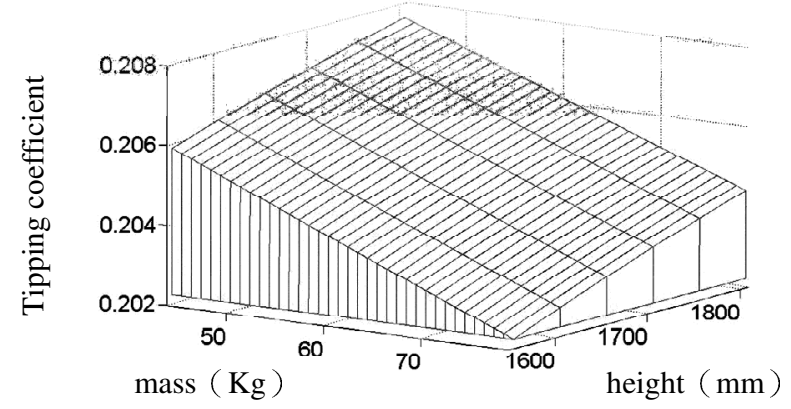

Figure 6. Relationships between tipping coefficient and height, mass. 


\section{Stability domains}

Assuming the coefficient of friction is sufficiently large and there is no slipping between human and deck, crews will move together with ocean platform. So the human body would have the same motion parameters with ocean platform. These motion parameters as $a_{x}, a_{y}$, and $\theta$ and $\ddot{\theta}$ can be regarded as the excitation that crews suffer from motions of ocean platform. In this paper, the model of human body considered has the height of $1700 \mathrm{~mm}$ and the mass of $60 \mathrm{~kg}$.

\subsection{Under the excitation of translational acceleration}

When the rotational motion is neglected, only translational motions with horizontal acceleration $a_{x}$ and vertical acceleration $a_{y}$ are considered. According to equation (10) and (11), two oblique straight lines could be obtained, as line 1 and line 2 in Figure 6 . The value of intersection points $C_{1}, C_{2}$ are $\pm \frac{w}{h} g$ on $\mathrm{x}$ axis. This is to say the stability domain is closely related to the tipping coefficient. If the tipping coefficient increases, the domains would be larger, as is shown by the dash lines. So large tipping coefficient is helpful for stability of human body.

When the vertical acceleration equals - $g$ the human body will be in the condition of weight loss and the contact loss between feet and floor as point $D$ in fig 6 . It is unstable whatever the horizon acceleration.

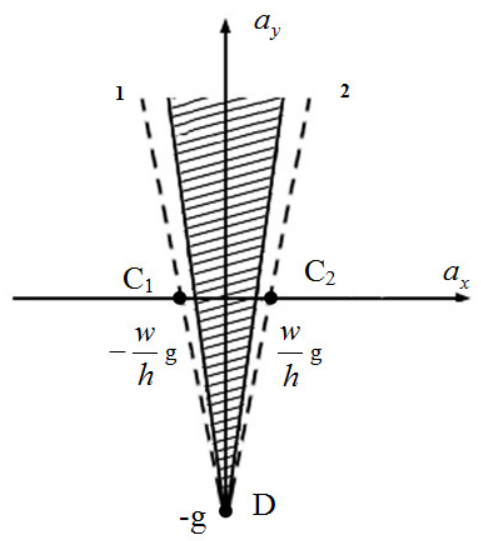

Figure 7. Stability domains under the excitation of translational acceleration.

\section{Conclusions}

In this paper, the postural stability for Chinese crew working on moving ocean platform is studied. Some human parameters as the height of COM and moment of inertia are calculated based on the standard of Chinese adults' anthropometric parameters. Effects on tipping coefficients of these parameters are investigated. Stability domain was obtained with excitation of translating accelerations. The work can be used as a guide to estimation the possibility of MII and to build an index to describe the stability degree of crews on moving platform.

\section{References}

1. A.H. Wertheim, Working in a moving environment, Ergonomics, 41 (12), 1845-1858 (1998) 
2. R. Graham, Motion-induced interruptions as ship operability criteria, Naval Engineers Journal 102 (2), 65-71 (1990)

3. P. Crossland and K. Rich, Validating a model of the effects of ship motion on postural stability, Proceedings of International Conference on Environmental Ergonomics, SanDiego (1998)

4. B.M. Haward, C.H. Lewis, and M.J. Griffin, Motions and crew responses on an offshore oil production and storage vessel, Applied Ergonomics, 40, 904-914 (2009)

5. N. Nawayseh and M.J. Griffin, Effect of frequency, magnitude and direction of translational and rotational oscillation on the postural stability of standing people, Journal of Sound and Vibration, 298 (6), 725-754 (2006)

6. J. Wedge and R.G. Langlois, Simulated the effects of ship motion on postural stability using articulated dynamic models, The Society for Modeling and Simulation, International Summer Computer Simulation Conference, SCSC Liophant \& SCS, Montreal, 20-23 July (2003)

7. B. Yao, Z. Chang, et al., Dynamic response of human body on offshore platform, Mechanical Design, 29 (3), 432-439 (2012) (In Chinese)

8. E. Nocerino, Human postural stability onboard ship as seakeeping criterion, Stance control model and procedure for validating it: a proposal[D], Università degli studi di Napoli Federico II (2010)

9. American Bureau of Shipping, Guide for Crew Habitability on offshore installations, Huston (2012)

10. National Standard Organization of China, GB/T 10000-1988, Human Dimensions for Chinese adults (1988)

11. National Standard Organization of China, GB/T 12985-1991, General rules of using percentiles of the body dimensions for product design (1991)

12. National Standard Organization of China, GB/T 17245-2004, Inertial parameters of adult human (2004) 